\title{
Polyethyleneimine is an effective permeabilizer of Gram-negative bacteria
}

\author{
Ilkka M. Helander, ${ }^{1}$ Hanna-Leena Alakomi, ${ }^{1}$ Kyosti Latva-Kala' \\ and Pertti Koski ${ }^{2}$
}

1 VTT Biotechnology and Food Research, PO Box 1501, FIN-02044 VTT, Espoo, Finland

2 Department of Vaccine Development, KTL, Mannerheimintie 166, FIN00300, Helsinki, Finland
Author for correspondence: Ilkka M. Helander. Tel: +358 94564452 . Fax: +35894552028. e-mail: Ilkka.Helander@vtt.fi

The effect of the polycation polyethyleneimine (PEI) on the permeability properties of the Gram-negative bacterial outer membrane was investigated using Escherichia coli, Pseudomonas aeruginosa and Salmonella typhimurium as target organisms. At concentrations of less than $20 \mu \mathrm{g} \mathrm{ml}^{-1}$, PEl increased the bacterial uptake of 1-N-phenylnaphthylamine, which is a hydrophobic probe whose quantum yield is greatly increased in a lipid environment, indicating increased hydrophobic permeation of the outer membrane by PEI. The effect of PEI was comparable to that brought about by the well-known permeabilizer EDTA. Permeabilization by PEI was retarded but not completely inhibited by millimolar concentrations of $\mathbf{M g C l}_{2}$. PEI also increased the susceptibility of the test species to the hydrophobic antibiotics clindamycin, erythromycin, fucidin, novobiocin and rifampicin, without being directly bactericidal. PEI sensitized the bacteria to the lytic action of the detergent SDS in assays where the bacteria were pretreated with PEI. In assays where PEI and SDS were simultaneously present, no sensitization was observed, indicating that PEI and SDS were inactivating each other. In addition, a sensitizing effect to the nonionic detergent Triton X-100 was observed for $P$. aeruginosa. In conclusion, PEI was shown to be a potent permeabilizer of the outer membrane of Gram-negative bacteria.

Keywords: polyethyleneimine, Gram-negative bacteria, outer membrane, lipopolysaccharide

\section{INTRODUCTION}

Polyethyleneimine (PEI) is a weakly basic aliphatic polymer which is polycationic due to the presence of primary, secondary and tertiary amino groups. PEIs are available in different molecular masses and forms, and they are widely utilized to flocculate cellular contaminants such as nucleic acids, lipids and debris from cellular homogenates to facilitate purification of soluble proteins (Cordes et al., 1990; Kirk \& Cowan, 1995; Milburn et al., 1990; Salt et al., 1995). Enzymic reactions in bioprocesses constitute another field where PEI is used, as an immobilizing agent for biocatalysts (Bahulekar et al., 1991), as a soluble carrier of enzymes (Cong et al., 1995), or in the formation of macrocyclic metal complexes mimicking metalloenzymes (Suh et al., 1991). A survey of patent data reveals that PEI is a

Abbreviations: NPN, 1-N-phenylnaphthylamine; OM, outer membrane; PEI, polyethyleneimine; PMBN, polymyxin B nonapeptide. common ingredient in a variety of formulations ranging from washing agents to packaging materials, and is also involved in microbicidal compositions. There are few data, however, concerning the microbicidal activity of PEI and its interaction with bacteria.

Certain polycationic substances such as polymyxin and its derivatives, polylysines and protamine are known to make the Gram-negative bacterial outer membrane (OM) permeable to solutes that normally are unable to penetrate it (for a review see Vaara, 1992). The OM is present in all Gram-negative bacteria, and due to the presence of the unique macromolecule LPS (for a review see Helander et al., 1996) in the outer leaflet of the membrane, it forms a permeability barrier against hydrophobic substances and macromolecules (Nikaido, 1989). For this reason Gram-negative bacteria exhibit higher resistance to detergents and hydrophobic antibiotics than do Gram-positive bacteria (Nikaido, 1989). Due to its polycationic nature, PEI could be expected to act as an OM-permeabilizing agent. We have therefore 
investigated the effect of PEI on different Gram-negative bacteria, focusing on phenomena associated with the permeability of the OM.

\section{METHODS}

Reagents. PEI (mean molecular mass $50 \mathrm{kDa}$ ), chicken egg white lysozyme (EC 3.2.1.17) and SDS were purchased from Sigma, and Triton X-100 from BDH.

Bacterial strains and growth conditions. The following strains were used: Escherichia coli ATCC 35150 (O157:H7), Pseudomonas aeruginosa ATCC 9027, Salmonella typhimurium ATCC 13311 and Salmonella enteritidis ATCC 13076. Unless otherwise stated, the growth medium in all liquid cultures was LB broth containing, per litre: $10 \mathrm{~g}$ Difco tryptone, $5 \mathrm{~g}$ Difco yeast extract, $5 \mathrm{~g} \mathrm{NaCl}, \mathrm{pH} \mathrm{7 \cdot 0}$. Cultivations were carried out at $37^{\circ} \mathrm{C}$ with shaking ( 200 r.p.m.). Growth was monitored by measuring $\mathrm{OD}_{630}$ with a Multiskan $\mathrm{MCC} / 340$ spectrophotometer (Labsystems).

Permeability assay based on the uptake of 1-N-phenylnaphthylamine (NPN). The permeability of viable bacteria was measured by the uptake of the fluorescent hydrophobic probe NPN essentially as described by Loh et al. (1984). Briefly, the bacteria were cultivated to $\mathrm{OD}_{630} 0 \cdot 5$, then the cells were deposited by centrifugation $(1000 \mathrm{~g}, 10 \mathrm{~min}$ at room temperature) and suspended in a similar volume of $5 \mathrm{mM}$ HEPES, pH 7.2. Samples $(1.35 \mathrm{ml})$ of this suspension were pipetted directly into the fluorometer cuvette, and fluorescence was monitored with a Shimadzu RF-5000 spectrophotometer operated at wavelengths of $350 \mathrm{~nm}$ (excitation) and $420 \mathrm{~nm}$ (emission) and slit widths of $5 \mathrm{~nm}$. After $30 \mathrm{~s}, \mathrm{NPN}(0.5 \mathrm{mM}$ in acetone) was added to a final concentration of $10 \mu \mathrm{M}$, and the resulting fluorescence was recorded. The test substance in aqueous solution was added, and the fluorescence again monitored. If desired, $\mathrm{MgCl}_{2}$ was added to the cell suspension before addition of NPN.

Bacteriolysis assay. The bacteriolytic effect was assayed on Nunclon microtitre plates (Nunc) by measuring the $\mathrm{OD}_{405}$ of bacterial cultures. First, the bacteria were grown to $\mathrm{OD}_{630} 0.5$, centrifuged as above, and suspended in a similar volume of $10 \mathrm{mM}$ HEPES $/ 50 \mathrm{mM} \mathrm{NaCl}$. This suspension was divided into two portions, one of which was supplemented with PEI at $10 \mu \mathrm{g} \mathrm{ml} \mathrm{m}^{-1}$. The suspensions were incubated at room temperature for $10 \mathrm{~min}$, centrifuged and cells resuspended in the buffer without PEI. Aliquots $\left(10^{8}\right.$ cells in $\left.100 \mu \mathrm{l}\right)$ in quadruplicate were then pipetted into microtitre wells, which already contained appropriate amounts of lysozyme, Triton $\mathrm{X}-100, \mathrm{SDS}$ or buffer only, and the $\mathrm{OD}_{405}$ monitored. The degree of cell lysis was determined from the $\mathrm{OD}_{405}$ values 4 min after the addition of cells. The value of the cell control without added lysozyme or detergent was taken as $100 \%$; strong lysis is thus indicated by low percentage. Each assay was performed three times.

Antibiotic susceptibility and growth inhibition tests. The susceptibility of bacterial cultures to hydrophobic antibiotics was tested with the agar diffusion method on Iso-Sensitest agar (Oxoid) without or with PEI supplementation (50-500 $\mu \mathrm{g}$ $\mathrm{ml}^{-1}$ ), using Neo-Sensitabs (erythromycin, novobiocin, clindamycin, fucidin and rifampicin; Rosco Diagnostica). The diameters of inhibition zones were measured after incubation of the plates at $37^{\circ} \mathrm{C}$ overnight.

Further susceptibility tests with PEI and combinations of PEI and novobiocin were performed as described by Skyttä \&
Mattila-Sandholm (1991) with an automated turbidimeter Bioscreen (Labsystems). A stock solution of novobiocin was prepared by dissolving $10 \mathrm{mg}$ novobiocin (sodium salt, Sigma) in $70 \%(\mathrm{v} / \mathrm{v})$ ethanol.The stock solution was further diluted $(1: 5)$ in distilled water, sterilized by filtration $(0.22 \mu \mathrm{m})$ and used the same day.

The antimicrobial activity of PEI and novobiocin against $S$. typhimurium ATCC 13311 was studied by dispensing $30 \mu \mathrm{l}$ of a $10^{-1}$ dilution from a culture that had been grown in IsoSensitest broth (ISB, Oxoid) for $5 \mathrm{~h}$ (200 r.p.m., $\left.37^{\circ} \mathrm{C}\right)$ to microtitre plate wells with $270 \mu$ l test medium (ISB supplemented with PEI or PEI and novobiocin) and incubated at $37^{\circ} \mathrm{C}$ for $24 \mathrm{~h}$. The tested PEI concentrations were 2 and $10 \mu \mathrm{g}$ $\mathrm{ml}^{-1}$; novobiocin was tested at 10,50 and $100 \mu \mathrm{g} \mathrm{ml}^{-1}$. In the control sample wells, the antimicrobial agent was replaced by an equal volume of sterile water or ethanol. All determinations were replicated five times and results were expressed as mean values. The area under the growth curve was used as a measure of microbial growth, and the percentage reduction of area was used to express the growth inhibition in the presence of the antimicrobials.

Assay of liberated lipid by fatty acid analysis. S. typhimurium ATCC 13311 was grown in LB broth to $\mathrm{OD}_{630} 0.6$, and the culture was divided into three $10 \mathrm{ml}$ portions, which were centrifuged to deposit the cells $\left(3000 \mathrm{~g}, 30 \mathrm{~min}, 25^{\circ} \mathrm{C}\right)$. The

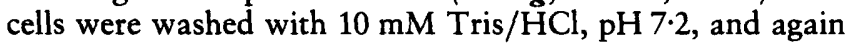
resuspended in $10 \mathrm{ml}$ of the same buffer, which contained either EDTA $(1 \mathrm{mM})$ or PEI $\left(10 \mu \mathrm{g} \mathrm{ml}^{-1}\right)$. The control sample was suspended in buffer only. After $10 \mathrm{~min}$ at $37^{\circ} \mathrm{C}$ the samples were centrifuged (11000 r.p.m. in an Eppendorf microfuge for $1 \mathrm{~min}$ at room temperature). Aliquots $(10 \mu \mathrm{l})$ of the cell-free supernatants were taken for SDS-PAGE analysis, the rest were freeze-dried and $105 \mu \mathrm{g}$ heptadecanoic acid methyl ester (Sigma) added to the dry samples, which were then subjected to fatty acid analysis. The samples were processed by saponification and methylation as described by Moore et al. (1994). The resulting fatty acid methyl esters were analysed with a HP5890 Series II (Hewlett-Packard) gas chromatograph equipped with a 7673A autosampler, a $25 \mathrm{~m} \times 0.2 \mathrm{~mm}$ Ultra-2 column (Hewlett-Packard) and a flame ionization detector operated at $300^{\circ} \mathrm{C}$. The injector temperature was $250^{\circ} \mathrm{C}$. The temperature programme was from $170^{\circ} \mathrm{C}$ to $260^{\circ} \mathrm{C}$ at $5^{\circ} \mathrm{C} \mathrm{min}^{-1}$ and then from $260^{\circ} \mathrm{C}$ to $300^{\circ} \mathrm{C}$ at $40^{\circ} \mathrm{C} \mathrm{min} \mathrm{m}^{-1}$. Helium was used as carrier gas with a velocity of $27 \mathrm{~cm} \mathrm{~s}^{-1}$. The split ratio was $1: 50$ and $1 \mu \mathrm{l}$ was injected.

Miscellaneous methods. SDS-PAGE was carried out by the Laemmli (1970) system, using the Novex Xcell II apparatus and pre-cast $12 \%$ acrylamide gels. Lysates for SDS-PAGE were prepared and treated with proteinase $\mathrm{K}$ (Sigma) according to Hitchcock \& Brown (1983).

\section{RESULTS}

\section{Permeabilization assay by NPN fluorescence}

NPN is a hydrophobic fluorescent probe whose quantum yield in hydrophobic environments is greatly increased as compared to hydrophilic environments, enabling its use in $O M$ permeabilization studies (Träuble \& Overath, 1973; Loh et al., 1984; Hancock et al., 1991; Tsuchido et al., 1989; Freer et al., 1996). We investigated the effect of PEI on NPN fluorescence in cell suspensions of $E$. coli, $P$. aeruginosa and $S$. typhimurium. Concentrations of PEI of up to $100 \mu \mathrm{g} \mathrm{ml}^{-1}$ in 


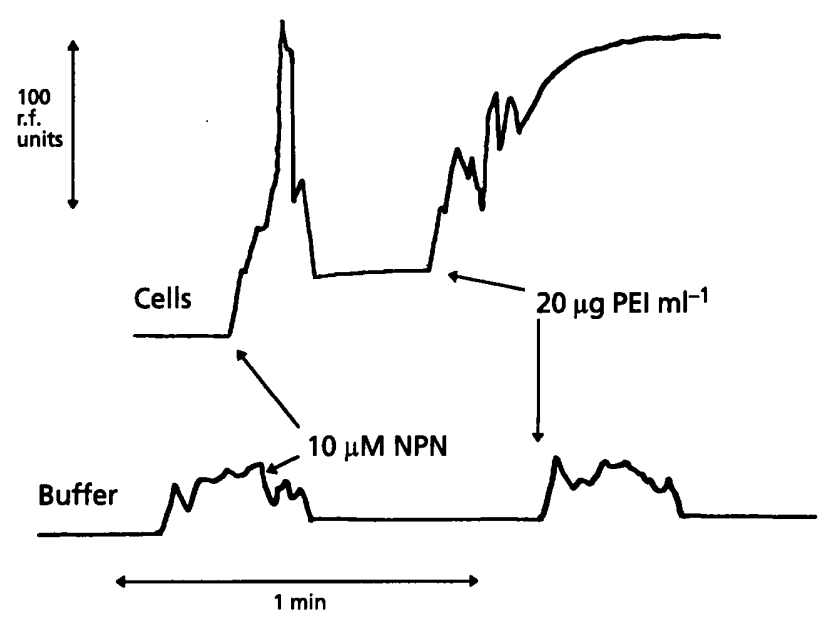

Fig. 1. Effect of addition of $10 \mu \mathrm{M} \mathrm{NPN}$ and $20 \mu \mathrm{g} \mathrm{PEI} \mathrm{ml}^{-1}$ into a suspension of $E$. coli (top) and into $5 \mathrm{mM}$ HEPES buffer without bacteria (bottom). Fluorescence at $420 \mathrm{~nm}$ (excitation wavelength, $350 \mathrm{~nm}$ ) was measured over time and expressed as relative fluorescence (r.f.) units. Sudden increases in fluorescence are due to opening of the cell chamber during addition of chemicals; the fluorescence values were obtained from stabilized curves.

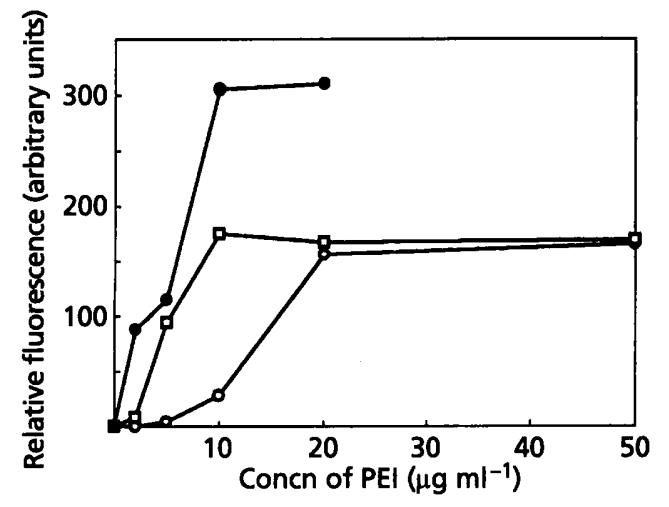

Fig. 2. Permeabilization of $E$. coli (O), P. aeruginosa $(O)$ and $S$. typhimurium $(\square)$ by PEI, assayed by NPN uptake.

5 mM HEPES buffer containing NPN $(10 \mu \mathrm{M})$ without bacteria caused no increase in fluorescence (Fig. 1). Fig. 1 also shows the result of a typical fluorescence assay in which NPN and PEI were sequentially added to a cell suspension of E. coli. Addition of NPN to the cell suspension always caused an increase in the fluorescence, and a prominent increase was observed after addition of PEI, exemplified in Fig. 1 by a concentration of $20 \mu \mathrm{g} \mathrm{ml}^{-1}$. With E. coli the fluorescence increase was dose-dependent so that the relative fluorescence values increased proportionately up to $20 \mu \mathrm{g} \mathrm{PEI} \mathrm{ml} \mathrm{I}^{-1}$ (Fig. 2), whereas with $P$. aeruginosa and $S$. typhimurium a saturation in the uptake of NPN was observed already at $10 \mu \mathrm{g} \mathrm{ml}^{-1}$ with cell suspensions adjusted to the same optical density as that of E. coli (Fig. 2). The highest levels of fluorescence were obtained with $P$. aeruginosa.

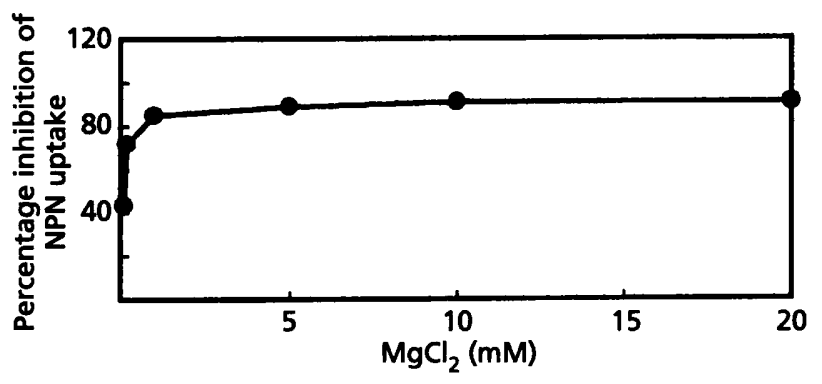

Fig. 3. Inhibition of PEl-induced permeabilization of $P$. aeruginosa by $\mathrm{MgCl}_{2}$ assayed by NPN uptake. The PEI concentration was $5 \mu \mathrm{g} \mathrm{ml}^{-1}$.

It was also noted that higher concentrations $\left(>10 \mu \mathrm{g} \mathrm{ml}^{-1}\right)$ of PEI resulted in the precipitation of bacterial cells, especially if the cultures had been centrifuged with refrigeration. Suspensions of $P$. aeruginosa were particularly prone to precipitation, and for this reason $P$. aeruginosa could not be tested with PEI concentrations higher than $20 \mu \mathrm{g} \mathrm{ml}^{-1}$.

The well-known permeabilizer EDTA, which destabilizes the OM by chelating divalent cations (reviewed by Vaara, 1992), was tested in parallel with PEI. With each of the three bacterial species mentioned above, $2 \mathrm{mM}$ EDTA caused fluorescence increases that did not differ from the maximal levels induced by PEI (data not shown), indicating that these two substances possess similar permeabilization potencies in the conditions employed.

Since divalent cations such as $\mathrm{Mg}^{2+}$ are known to inhibit the action of many OM permeabilizers that act by either chelating or replacing cations in the OM (Vaara, 1992), we tested whether $\mathrm{Mg}^{2+}$ affects the activity of PEI. Fig. 3 shows the inhibitory effect of added $\mathrm{MgCl}_{2}$ on the NPN uptake in the presence of $5 \mu \mathrm{g}$ PEI $\mathrm{ml}^{-1}$ by $P$. aeruginosa. Even at submillimolar concentrations $\mathrm{Mg}^{2+}$ had an inhibitory effect on NPN uptake, the maximum inhibition remaining at $90 \%$ within the concentration range tested. NPN uptake in the presence of $\mathrm{Mg}^{2+}$ reached a constant value after a period of typically less than $30 \mathrm{~s}$. For PEI-induced NPN uptake by $S$. typhimurium and $E$. coli, the inhibition by $\mathrm{Mg}^{2+}$ was less prominent and exhibited different kinetics from that observed with $P$. aeruginosa, in that $\mathrm{Mg}^{2+}$ only slowed the uptake of NPN. Thus in the presence of $\mathrm{Mg}^{2+}$ a retarded but constantly increasing fluorescence was observed, as shown in Fig. 4 for E. coli with $20 \mu \mathrm{g}$ PEI $\mathrm{ml}^{-1}$ and $1 \mathrm{mM} \mathrm{MgCl}$. For $S$. typhimurium, tested with $5 \mu \mathrm{g}$ PEI ml $\mathrm{m}^{-1}, \mathrm{Mg}^{2+}$ exhibited a clear-cut inhibitory effect in concentrations higher than $1 \mathrm{mM}$.

\section{Effect of PEI on antibiotic susceptibility}

A sensitizing effect to hydrophobic antibiotics is one of the indications of OM-permeabilizing action (Vaara, 1992). We tested the susceptibility of E. coli, P. aeru- 


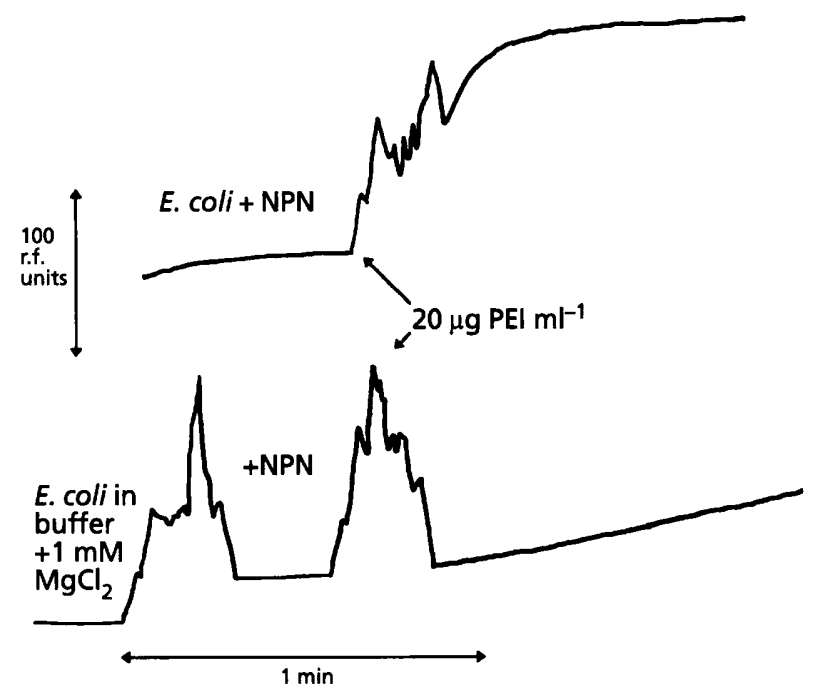

Fig. 4. Uptake of NPN by suspensions of $E$. coli induced by $20 \mu \mathrm{g} \mathrm{ml}^{-1} \mathrm{PEI}$ in the absence (top) and presence (bottom) of $1 \mathrm{mM} \mathrm{MgCl} 2$.

Table 1. Effect of PEI on the susceptibility of $E$. coli, $P$. aeruginosa and $S$. typhimurium to selected antibiotics determined by the agar diffusion method

\begin{tabular}{|lrrrr|}
\hline Antibiotic & \multicolumn{3}{c|}{$\begin{array}{c}\text { Diameter of inhibition zone } \\
\text { (mm) on plated cultures }\end{array}$} \\
\cline { 2 - 5 } & \multicolumn{4}{c}{ PEI concn $\left(\boldsymbol{\mu g ~ m}^{-\mathbf{1}}\right)$ : } \\
& $\mathbf{0}$ & $\mathbf{5 0}$ & $\mathbf{2 0 0}$ & $\mathbf{5 0 0}$ \\
\hline E. coli & & & & \\
Erythromycin & 17 & 18 & 19 & 18 \\
Novobiocin & 12 & 15 & 25 & 27 \\
Clindamycin & 0 & 17 & 17 & 20 \\
Fucidin & 0 & 0 & 20 & 25 \\
Rifampicin & 23 & 25 & 25 & 30 \\
P. aeruginosa & & & & \\
Erythromycin & 19 & 20 & 17 & 23 \\
Novobiocin & 0 & 0 & 20 & 45 \\
Clindamycin & 0 & 0 & 0 & 15 \\
Fucidin & 0 & 0 & 0 & 22 \\
Rifampicin & 17 & 19 & 19 & 39 \\
S. typhimurium & & & & \\
Erythromycin & 16 & 16 & 21 & 24 \\
Novobiocin & 0 & 15 & 22 & 30 \\
Clindamycin & 0 & 0 & 16 & 16 \\
Fucidin & 0 & 0 & 13 & 21 \\
Rifampicin & 22 & 22 & 24 & 30 \\
\hline
\end{tabular}

ginosa and S. typhimurium to a set of hydrophobic antibiotics (erythromycin, novobiocin, clindamycin, fucidin and rifampicin) by the agar diffusion method on plates containing different concentrations of PEI. The results are summarized in Table 1 , which shows that PEI
Table 2. Synergistic inhibition of growth of $S$. typhimurium by PEI and novobiocin

The results are means of five determinations $\pm S D$.

\begin{tabular}{|cccc|}
\hline $\begin{array}{l}\text { Novobiocin } \\
\text { concn } \\
\left(\mu \mathrm{g} \mathrm{m}^{-1}\right)\end{array}$ & \multicolumn{3}{c|}{ Growth inhibition (\%) in $24 \mathrm{~h}$} \\
\cline { 2 - 4 } & \multicolumn{3}{c|}{ PEI concn $\left(\mu \mathrm{g} \mathrm{ml}^{-1}\right):$} \\
& 0 & 2 & 10 \\
\hline 0 & 0 & $4 \cdot 8 \pm 4 \cdot 5$ & $8 \cdot 8 \pm 4 \cdot 3$ \\
10 & 0 & 0 & $40 \cdot 6 \pm 3.0$ \\
30 & 0 & 0 & $61 \cdot 2 \pm 0.9$ \\
100 & $1 \cdot 7 \pm 1 \cdot 5$ & $22 \cdot 6 \pm 9.9$ & $63 \cdot 4 \pm 6 \cdot 8$ \\
\hline
\end{tabular}

induced clear-cut susceptibility to novobiocin, clindamycin and fucidin, these antibiotics being ineffective towards the test bacteria in the absence of PEI. However, on agar plates much larger concentrations ( $>50 \mu \mathrm{g} \mathrm{ml}^{-1}$ ) were required for sensitization to antibiotics than for NPN uptake in suspension $\left(10 \mu \mathrm{g} \mathrm{ml}^{-1}\right)$. There was only weak, if any, sensitizing action for erythromycin, and for rifampicin the sensitizing effect was observed at $500 \mu \mathrm{g}$ PEI ml$l^{-1}$, most clearly for $P$. aeruginosa.

The effect of PEI on growth of S. typhimurium was also tested using the automated turbidimeter Bioscreen, where a definite sensitizing effect to novobiocin was observed (Table 2). In this experiment, PEI alone at concentrations of 2 and $10 \mu \mathrm{g} \mathrm{ml}^{-1}$ brought about only negligible growth inhibition of $S$. typhimurium. Essentially similar results were obtained for $E$. coli and $P$. aeruginosa with combinations of PEI and novobiocin. Furthermore, in Bioscreen assays concentrations of up to $500 \mu \mathrm{g}$ PEI ml ${ }^{-1}$ alone caused no significant growth inhibition of E. coli, $P$. aeruginosa or S. typhimurium.

\section{Sensitization to lytic action of lysozyme and detergents}

Permeabilization of the $O M$ is also manifested as an increased susceptibility to the bacteriolytic action of detergents and, in some cases, lysozyme (Vaara, 1992). We therefore tested the effect of PEI on the action of lysozyme and the detergents SDS and Triton X-100 on E. coli, $P$. aeruginosa and $S$. typhimurium. In these assays, cells were pretreated with PEI and subsequently washed so that PEI was not present in the assay medium. The results are shown in Table 3 . Lysozyme $\left(10 \mu \mathrm{g} \mathrm{ml}^{-1}\right)$ exhibited no lytic activity towards the untreated cells of any of the three species. With $P$. aeruginosa a weak lysis was observed in the PEI-treated culture, whereas cultures of E. coli and S. typhimurium remained unaffected. Only $P$. aeruginosa was sensitized by PEI to Triton X100. All of the test species were, however, strongly sensitized to SDS. Experiments with S. typhimurium were also carried out in which PEI was present together with lysozyme, SDS or Triton X-100 in the microtitre plate wells. In these tests no sensitization to SDS was 


\section{Table 3. Effect of PEI on bacteriolysis}

The degree of cell lysis was determined from the $\mathrm{OD}_{405}$ values $4 \mathrm{~min}$ after the addition of cells. The value of the cell control without added lysozyme or detergent was taken as $100 \%$; strong lysis is thus indicated by a low percentage. Each assay was performed three times with (+PEI) or without

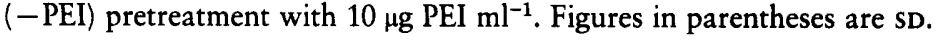

\begin{tabular}{|c|c|c|c|c|c|c|}
\hline \multirow[t]{3}{*}{ Lytic agent } & \multicolumn{6}{|c|}{ Relative turbidity (\%) } \\
\hline & \multicolumn{2}{|c|}{ E. coli } & \multicolumn{2}{|c|}{ P. aeruginosa } & \multicolumn{2}{|c|}{ S. typhimurium } \\
\hline & $-\mathrm{PEI}$ & + PEI & $-\mathrm{PEI}$ & $+\mathbf{P E I}$ & $-\mathrm{PEI}$ & + PEI \\
\hline Lysozyme $\left(10 \mu \mathrm{g} \mathrm{ml}^{-1}\right)$ & $100(1)$ & $101(1)$ & $100(2)$ & $92(7)$ & $100(2)$ & $101(3)$ \\
\hline $\operatorname{SDS}\langle 0 \cdot 1 \%, w / v\rangle$ & $100(2)$ & $38(8)$ & $102(3)$ & $31(19)$ & $64(2)$ & $23(2)$ \\
\hline $\operatorname{SDS}(1 \%, \mathrm{w} / \mathrm{v})$ & $92(1)$ & $35(10)$ & $93(4)$ & $24(18)$ & $21(4)$ & $22(2)$ \\
\hline Triton X-100 $(0.1 \%, \mathrm{w} / \mathrm{v})$ & $102(1)$ & $102(1)$ & $100(3)$ & $78(6)$ & $101(2)$ & $101(1)$ \\
\hline Triton X-100 (1\%, w/v) & $92(1)$ & $92(1)$ & $95(5)$ & $62(12)$ & $88(1)$ & $89(3)$ \\
\hline
\end{tabular}

Table 4. Assay of fatty acids derived from cell-free supernatants of S. typhimurium cultures treated with EDTA or PEI

\begin{tabular}{|c|c|c|c|}
\hline \multirow[t]{2}{*}{ Fatty acid } & \multicolumn{3}{|c|}{ Concn $\left[\mu \mathrm{g}(\mathrm{ml} \text { cell-free supernatant })^{-1}\right]$} \\
\hline & Untreated & + EDTA (1 mM) & + PEI $\left(10 \mu \mathrm{g} \mathrm{ml}^{-1}\right)$ \\
\hline $12: 0$ & 0.09 & $0 \cdot 37$ & 0.05 \\
\hline 14:0 & $0 \cdot 11$ & 0.60 & $<0.02$ \\
\hline $16: 0$ & 0.36 & 1.53 & $0 \cdot 10$ \\
\hline $18: 0$ & $<0.02$ & $<0.02$ & $<0.02$ \\
\hline $17: 0$ cyc* $^{*}$ & $<0.02$ & 0.20 & $<0.02$ \\
\hline $16: 1$ & $0 \cdot 18$ & 0.83 & $<0.02$ \\
\hline $18: 1$ & $0 \cdot 19$ & $1 \cdot 01$ & $<0.02$ \\
\hline $14: 0(3-\mathrm{OH})$ & $0 \cdot 22$ & $1 \cdot 28$ & $<0.02$ \\
\hline $\begin{array}{l}\text { Sum of quantified } \\
\text { fatty acids }\end{array}$ & $1 \cdot 15$ & $5 \cdot 82$ & $0 \cdot 15$ \\
\hline
\end{tabular}

* cyc, cyclopropane.

observed. Instead, a weak synergistic effect was noted for combinations of PEI with either lysozyme or Triton $\mathrm{X}-100$ (data not shown).

\section{Does PEI liberate LPS?}

Permeabilizing agents such as chelators and certain polycations release LPS from the OM, whereas others such as polymyxin $\mathrm{B}$ nonapeptide (PMBN) cause OM permeabilization without significant liberation of LPS (Vaara, 1992). To investigate the mechanism of action of PEI upon S. typhimurium, we treated bacterial suspensions with PEI and in parallel with EDTA in Tris buffer, and analysed the material remaining in the cellfree supernatants by fatty acid analysis to reveal LPSspecific fatty acids indicating liberation of LPS from the OM. Table 4 shows the amounts of fatty acids found in the supernatants after saponification and methylation. Fatty acids typical of S. typhimurium cells were present in the control sample, indicating some release of membrane components to the medium. In the EDTAtreated sample the total amount of fatty acids was several times higher, and the proportion of the LPSspecific 3-hydroxytetradecanoic acid was approximately six times larger than that found in the control sample. This indicates that EDTA had liberated LPS from the cells. In the PEI-treated sample, the amount of all fatty acids was very low, showing that the supernatants contained no detectable LPS. This was confirmed by SDS-PAGE, as no LPS could be visualized in gels by silver staining in lanes loaded with proteinase-K-treated supernatants of PEI supernatants. Instead, samples of EDTA supernatants revealed ladder-like patterns typical of smooth-type LPS (not shown). It is apparent from this experiment that upon treatment with PEI no LPS was liberated from the cells in a soluble form. The possibility still exists, however, that PEI released LPS from the OM, but due to the water-insoluble nature of the PEI-LPS 
complex it could not be detected in the cell-free supernatant.

\section{DISCUSSION}

On the basis of our results it can be concluded that even at sub-inhibitory concentrations PEI makes Gramnegative bacteria permeable to hydrophobic probes such as antibiotics and NPN. Sensitization of Gram-negative bacteria to hydrophobic antibiotics is a property shared by all polycationic permeabilizers such as deacylated polymyxin derivatives containing a cyclic heptapeptide, e.g. PMBN (reviewed by Vaara, 1992), polylysine and protamine (Vaara \& Vaara, 1983a). Of these agents, the latter two polycations release considerable proportions of LPS from the OM, whereas PMBN acts in a different manner, binding to LPS but not releasing it to the medium (Vaara \& Vaara, 1983b). Bacteria are known not to be sensitized to the anionic detergent SDS when tested together with the above permeabilizers (Vaara \& Vaara, 1983a), as was also shown for PEI. It can be assumed that the large polycation PEI interacts electrostatically with SDS and probably sequesters it to an ineffective form. However, PEI did strongly sensitize all test bacteria to SDS in our assay in which cells were pretreated with PEI. This shows that PEI was able to disorganize the $\mathrm{OM}$ not only transiently but in an irreversible manner in these conditions.

No sensitization to lysozyme was observed with PEI, in accordance with the similar inability of other polycationic permeabilizers. The permeation of lysozyme apparently requires extensive disorganization in the $O M$, such as that resulting from the massive loss of LPS from the OM by EDTA, as first shown by Leive (1965). We could not demonstrate liberation of LPS from $S$. typhimurium by PEI. PEI is known to bind to and precipitate various cellular materials such as lipids and nucleic acids (Salt et al., 1995). Since LPS molecules are normally anionic due to the presence of phosphate and carboxyl groups (Helander et al., 1996), PEI can be expected to bind strongly to LPS, forming insoluble complexes that make it impossible to measure LPS liberation by investigating cell-free supernatants after centrifugation of PEI-treated cultures. At present it seems most probable that PEI disorganizes the OM by intercalating in the LPS layer similarly to PMBN. The interactions of PEI and LPS certainly warrant further study, and since PEIs are available in different molecular sizes and forms, it would be of interest to determine their properties with respect to interactions with bacterial surface components. It would also be interesting to test the ability of PEI to permeabilize Gram-positive bacteria and eukaryotic cells; in fact PEI has been shown to affect neutrophil plasma membranes, resulting in the leakage of enzymes from the cells (Elferink, 1991).

Although a potent permeabilizer, PEI did not inhibit the growth of $E$. coli, $P$. aeruginosa or $S$. typhimurium to any significant extent. This is in agreement with Tashiro (1992), who showed that PEI600 had a small bacteriostatic effect on E. coli and P. aeruginosa.
By virtue of its permeability-increasing properties, PEI can be expected to act as a vehicle for experimental drug delivery into cells, and as a potentiating agent for antibacterial agents that are normally excluded by Gram-negative bacteria. Although PEI has a good safety record, further studies will be required to evaluate its applicability in areas such as disinfection and food protection.

\section{ACKNOWLEDGEMENTS}

We thank Päivi Lepistö and Ulla Vornamo for excellent technical assistance. This work was supported by the NISIN $^{\text {PLUS }}$ project (FAIR-CT96-1148).

\section{REFERENCES}

Bahulekar, R., Ayyangar, N. R. \& Ponrathnam, S. (1991). Polyethyleneimine in immobilization of biocatalysts. Enzyme Microb Technol 13, 858-868.

Cong, L., Kaul, R., Dissing, U. \& Mattiasson, B. (1995). A model study on Eudragit and polyethyleneimine as soluble carriers of $\alpha$ amylase for repeated hydrolysis of starch. J Biotechnol 42, 75-84.

Cordes, R. M., Sims, W. B. \& Glatz, C. E. (1990). Precipitation of nucleic acids with poly(ethyleneimine). Biotechnol Prog 6, 283-285.

Elferink, J. G. R. (1991). Changes of plasma membrane permeability in neutrophils treated with polycations. Inflammation 15, 103-115.

Freer, E., Moreno, E., Moriyón, l., Pizarro-Cerdá, J., Weintraub, A. \& Gorvel, J.-P. (1996). Brucella-Salmonella lipopolysaccharide chimeras are less permeable to hydrophobic probes and more sensitive to cationic peptides and EDTA than are their native Brucella sp. counterparts. J Bacteriol 178, 5867-5876.

Hancock, R. E. W., Farmer, S. W., Li, Z. \& Poole, K. (1991). Interaction of aminoglycosides with the outer membranes and purified lipopolysaccharide and OmpF porin of Escherichia coli. Antimicrob Agents Chemother 35, 1309-1314.

Helander, I. M., Makela, P. H., Westphal, O. \& Rietschel, E. T. (1996). Lipopolysaccharides. In Encyclopedia of Molecular Biology and Molecular Medicine, vol. 3, pp. 462-471. Edited by R. A. Meyers. Weinheim: VCH.

Hitchcock, P. J. \& Brown, T. M. (1983). Morphological heterogeneity among Salmonella lipopolysaccharide chemotypes in silver-stained polyacrylamide gels. J Bacteriol 154, 269-277.

Kirk, N. \& Cowan, D. (1995). Optimising the recovery of recombinant thermostable proteins expressed in mesophilic hosts. J Biotechnol 42, 177-184.

Laemmli, U. K. (1970). Cleavage of structural proteins during the assembly of the head of bacteriophage T4. Nature 227, 680-685. Leive, L. (1965). Release of lipopolysaccharide by EDTA treatment of E. coli. Biochem Biophys Res Commun 21, 290-296.

Loh, B., Grant, C. \& Hancock, R. E. W. (1984). Use of the fluorescent probe $1-N$-phenylnaphthylamine to study the interactions of aminoglycoside antibiotics with the outer membrane of Pseudomonas aeruginosa. Antimicrob Agents Chemother 26, 546-551.

Milburn, P., Bonnerjea, J., Hoare, M. \& Dunnill, P. (1990). Selective flocculation of nucleic acids, lipids, and colloidal particles from a yeast cell homogenate by polyethyleneimine, and its scale-up. Enzyme Microb Technol 12, 527-532.

Moore, L. V. H., Bourne, D. M. \& Moore, W. E. C. (1994). Comparative distribution and taxonomic value of cellular fatty acids 
in thirty-three genera of anaerobic Gram-negative bacilli. Int J Syst Bacteriol 44, 338-347.

Nikaido, H. (1989). Outer membrane barrier as a mechanism of antimicrobial resistance. Antimicrob Agents Chemother 33, 1831-1836.

Salt, D. E., Hay, S., Thomas, O. R. T., Hoare, M. \& Dunnill, P. (1995). Selective flocculation of cellular contaminants from soluble proteins using polyethyleneimine: a study of several organisms and polymer molecular weights. Enzyme Microb Technol 17, 107-113.

Skyttä, E. \& Mattila-Sandholm, T. (1991). A quantitative method for assessing bacteriocins and other food antimicrobials by automated turbidometry. J Microbiol Methods 14, 77-88.

Suh, J., Cho, Y. \& Lee, K. J. (1991). Macrocyclic metal complexes built on polyethyleneimine. J Am Chem Soc 113, 4198-4202.

Tashiro, T. (1992). Removal of bacteria from water by systems based on insoluble polystyrene-polyethylenimine. J Appl Polym Sci 46, 899-907.
Träuble, H. \& Overath, P. (1973). The structure of Escherichia coli membranes studied by fluorescence measurements of lipid phase transitions. Biochim Biophys Acta 307, 491-512.

Tsuchido, T., Aoki, I. \& Takano, M. (1989). Interaction of the fluorescent dye 1-N-phenylnaphthylamine with Escherichia coli cells during heat stress and recovery from heat stress. J Gen Microbiol 135, 1941-1947.

Vaara, M. (1992). Agents that increase the permeability of the outer membrane. Microbiol Rev 56, 395-411.

Vaara, M. \& Vaara, T. (1983a). Polycations sensitize enteric bacteria to antibiotics. Antimicrob Agents Chemother 24, 107-113.

Vaara, M. \& Vaara, T. (1983b). Polycations as outer membranedisorganizing agents. Antimicrob Agents Chemother 24, 114-122.

Received 16 May 1997; revised 27 June 1997; accepted 2 July 1997. 